\title{
The Insulin-Like Growth Factor Receptor I Promotes Motility and Invasion of Bladder Cancer Cells through Akt- and Mitogen-Activated Protein Kinase- Dependent Activation of Paxillin
}

\author{
David Metalli, ${ }^{+}{ }^{\dagger}$ Francesca Lovat, ${ }^{*}$ \\ Farida Tripodi, ${ }^{* \dagger}$ Marco Genua, ${ }^{*}$ Shi-Qiong Xu, ${ }^{*}$ \\ Michela Spinelli, ${ }^{*}$ Lilia Alberghina, ${ }^{\dagger}$ \\ Marco Vanoni, ${ }^{\dagger}$ Raffaele Baffa, ${ }^{*}$ \\ Leonard G. Gomella, ${ }^{* \ddagger}$ Renato V. Iozzo, ${ }^{\S \pi}$ \\ and Andrea Morrione ${ }^{\star \ddagger}$

\begin{abstract}
From the Department of Urology,* the Endocrine Mechanisms and Hormone Action Program, ${ }^{\ddagger}$ the Cancer Cell Biology and Signaling Program, ${ }^{\S}$ and the Department of Pathology, Anatomy,

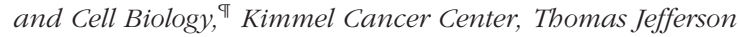
University, Philadelphia, Pennsylvania; and the Department of Biotechnology and Biosciences, ${ }^{\dagger}$ University of Milano-Bicocca, Milano, Italy
\end{abstract}

The insulin-like growth factor receptor I (IGF-IR) plays an essential role in transformation by promoting cell growth and protecting cancer cells from apoptosis. Aberrant IGF-IR signaling is implicated in several types of tumors, including carcinomas of the lung, breast, prostate, pancreas, liver, and colon. However, the contribution of the IGF-IR to the development of the transformed phenotype in urothelial cells has not been clearly established. In this study we demonstrated that the IGF-IR is overexpressed in invasive bladder cancer tissues compared with nonmalignant controls. We have investigated the role of the IGF-IR in bladder cancer by using urothelial carcinoma-derived 5637 and T24 cells. Although activation of the IGF-IR did not appreciably affect their growth, it did promote migration and stimulate in vitro wound closure and invasion. These effects required the activation of the Akt and Mitogen-activated protein kinase (MAPK) pathways as well as IGF-I-induced Akt- and MAPK-dependent phosphorylation of paxillin, which relocated at dynamic focal adhesions and was necessary for promoting motility in bladder cancer cells. Our results provide the first evidence for a role of the IGF-IR in motility and invasion of bladder cancer cells and support the hypothesis that the IGF-IR may play a critical role in the establishment of the invasive phenotype in urothelial neoplasia. Thus, the IGF-IR may also serve as a novel biomarker for bladder cancer. (Am J Patbol 2010, 176:2997-3006; DOI: 10.2353/ajpath.2010.090904)

Bladder cancer is a major epidemiological problem, whose incidence continues to rise each year. The most recent cancer statistic ${ }^{1}$ has estimated 68,810 new cases with 14,100 estimated deaths in the United States.

Bladder tumors show widely differing histopathological and clinical behavior, ${ }^{2}$ and this is a key problem in the management of bladder tumors. The majority of bladder tumors $(\sim 70 \%)$ are low-grade noninvasive papillary tumors that do not penetrate the epithelial basement membrane (Ta stage). The remainder comprises tumors that have penetrated the basement membrane but not invaded the muscle layer of the bladder wall (T1 stage) and muscle-invasive tumors (T2, T3, and T4 stages). ${ }^{3}$

The insulin-like growth factor receptor I (IGF-IR) plays a critical role in cell growth both in vitro ${ }^{4}$ and in vivo. ${ }^{5}$ Mice with targeted ablation of the IGF-IR gene have severe growth retardation, being only $45 \%$ the size of wild-type

Supported by the Benjamin Perkins Bladder Cancer Fund, the Martin Greitzer Fund, and National Institutes of Health grants RO1 DK068419 (A.M.), RO1 CA39481, and RO1 CA047282 (R.V.I.). D.M. was partially supported by Fondazione Fratelli Confalonieri.

D.M. and F.L. contributed equally to this work.

Accepted for publication February 9, 2010

Supplemental material for this article can be found on http://ajp. amjpathol.org.

A guest editor acted as editor-in-chief for this article. No person at Thomas Jefferson University was involved in the peer review process or final disposition for this article.

Current address of R.B.: Medimmune, Gaithersburg, MD.

Address reprint requests to Andrea Morrione, Ph.D., Department of Urology, Endocrine Mechanisms and Hormone Action Program, Kimmel Cancer Center, Thomas Jefferson University, 233 South 10th St, BLSB Room 620, Philadelphia, PA 19107. E-mail: andrea.morrione@jefferson.edu. 
littermates, and die shortly after birth primarily due to respiratory failure. ${ }^{6,7}$ These data suggest that the IGF-I/ IGF-IR axis is critical for normal growth.

The importance of the IGF-IR in transformation was initially suggested by experiments performed with cells derived from the IGF-IR-deficient mice. These cells were refractory to transformation induced by several tumorigenic agents (viral oncogenes, including Ras, SV40 large T Ag and overexpressed platelet-derived growth factor receptor and epidermal growth factor receptor, and various chemical agents) but were transformed when the IGF-IR was re-expressed. ${ }^{8,9}$ In vitro experiments on tumor cell lines and epidemiological studies have confirmed that activation of the IGF-IR is involved in the development of many common neoplastic diseases, including carcinomas of lung, prostate, pancreas, liver, colon, and breast. ${ }^{8,10,11}$ The transforming capability of the IGF-IR most likely depends on its ability to protect cancer cells from apoptosis. ${ }^{12-15}$

The IGF-IR has now become a very attractive target for cancer therapy, and in fact antibodies against the IGF-IR are currently in phase I clinical trials. ${ }^{16,17}$ Whether the IGF-IR contributes to the transforming phenotype of urothelial cells has not been clearly established, but recent data suggest that the IGF-IR is overexpressed in bladder cancer. ${ }^{18}$

In this study, using 5637 and T24 urothelial carcinomaderived cells, we established that activation of the IGF-IR plays a critical role in bladder cancer by promoting migration, wound healing, and invasion of cancer urothelial cells. We have also characterized the mechanism of action of the IGF-IR in cancer urothelial cells and showed that IGF-IR-dependent cell motility and invasion required the activation of the Akt and MAPK pathway and Akt- and Extracellular-signal-related kinase 1 (ERK)-dependent activation of paxillin.

Collectively, these results provide novel information toward a better understanding of the mechanisms that regulate tumor formation in bladder cancer at the cellular and biochemical level and suggest that the IGF-IR may be critical for bladder cancer.

\section{Materials and Methods}

\section{Immunohistochemical Detection of the IGF-IR in Normal and Cancer Bladder Tissue Specimens}

Immunohistochemical analysis of IGF-IR levels in bladder tissues was performed as previously described. ${ }^{19}$ Formalin-fixed paraffin-embedded sections from five invasive (T3/T4) urothelial cell carcinomas and adjacent normal tissues were obtained from the Pathology Tissue Bank of Thomas Jefferson University (Philadelphia, PA). Informed consent to use excess pathological specimens for research purposes was obtained from all five patients. Slides were incubated with 1:500 dilution of an anti-human IGF-IR polyclonal antibody (C-20; Santa Cruz Biotechnology, Santa Cruz, CA). The specificity of this antibody has been previously validated. ${ }^{18}$

\section{Cells, Growth, and Migration Assays}

Invasive urothelial carcinoma-derived human 5637 and T24 cell lines were obtained from the American Type Culture Collection (Manassas, VA). Cells were maintained in RPMI medium supplemented with $10 \%$ fetal bovine serum. Serum-free medium (SFM) is Dulbecco's modified Eagle's medium supplemented with $0.1 \%$ bovine serum albumin and $50 \mu \mathrm{g} / \mathrm{ml}$ of transferrin (Sigma-Aldrich Corp., St. Louis, MO). Cells were plated in duplicate at a density of $3 \times 10^{4}$ cells/35-mm plates in serum-supplemented medium. After 24 hours, cells were transferred to SFM or SFM supplemented with $50 \mathrm{ng} / \mathrm{ml}$ of IGF-I (Calbiochem, San Diego, CA). Cells were counted after 48 and 72 hours with a hemocytometer.

Migration experiments were performed by using HTS FluoroBloks inserts (Becton Dickinson, Franklin Lakes, $\mathrm{NJ})$ as previously described. ${ }^{19}$ Membranes were mounted on a slide, and migrated cells were counted and photographed with a Zeiss Axiovert 200M cell live microscope (Carl Zeiss SMT, Inc., Thornwood, NY) at the Kimmel Cancer Center Bioimaging Facility.

\section{Inhibitors}

Specific inhibitors for the Akt, LY294002, ERK1/2, or U0126 pathways (Calbiochem) were used at a concentration of $20 \mu \mathrm{mol} / \mathrm{L}$ and $10 \mu \mathrm{mol} / \mathrm{L}$, respectively, as described. ${ }^{20,21}$ For immunoblots, cells were pre-incubated with the inhibitors for 1 hour and then stimulated with IGF-I alone or IGF-I supplemented with the inhibitors.

\section{Wound Healing and Invasion Assays}

Cells were seeded onto $35-\mathrm{mm}$ plates in serum-containing medium until subconfluence and serum-starved for 24 hours. The plates were then scratched with a thin disposable tip to generate a wound in the cells monolayer ${ }^{20,21}$ and incubated in SFM or SFM supplemented with IGF-I. Cells were analyzed and photographed after 24 hours with a Zeiss Axiovert 200M cell live microscope by using the Metamorph Image Acquisition and Analysis software (Universal Imaging, Sunnyvale, CA).

Cell invasion through a 3D-extracellular matrix was assessed by a Matrigel invasion assay by using BD Matrigel Invasion Chambers (BD Biocoat, San Jose, CA). ${ }^{19}$ After 24 hours (5637) or 8 hours (T24), filters were washed, fixed, and stained with Coomassie Brilliant Blue (Sigma-Aldrich Corp.). Cells that had invaded to the lower surface of the filter were counted under the microscope.

As recently described, 3D evasion assays of T24 cells (5000/drop) in Matrigel drops (6 mg/ml; Becton Dickinson) were performed as recently described. ${ }^{19,22}$ Cell motility was analyzed by microscopy. Images were collected by using a digital camera (Nikon, Melville, NY) and cells outside each drop (five drops/cell line/experiment) were counted. 


\section{Detection of Activated Signaling Pathways}

Serum-starved cells were stimulated with IGF-I $(50 \mathrm{ng} / \mathrm{ml})$ for 10, 30, and 120 minutes. The activation of p90RSK, Akt, ERK1/2, and S6 ribosomal protein was analyzed by Western immunoblot by using the PathScan Multiplex Western Cocktail I (Cell Signaling Technology, Beverly, MA). EIF4E protein was used as control to monitor the loading of the samples.

Paxillin phosphorylation was detected by immunoblot either after immunoprecipitation with anti-paxillin monoclonal antibodies (BD Biosciences, San Jose, CA) or in cell lysates by using anti-phospho-paxillin antibodies. P-Y31 is from Genetex (Irvine, CA). P-S126 is from Biosource International (Camarillo, CA). P-S178 is from Calbiochem. Pan anti-phospho-tyrosine-horseradish peroxidase-conjugated monoclonal antibodies are from BD Transduction Laboratories (Lexington, $\mathrm{KY}$ ). Densitometric analysis was performed by using the ImageJ program (NIH, rsbweb.nih.gov/ij/, last accessed November 20, 2009).

\section{Small-Interfering RNA-Mediated Gene Silencing}

To silence the IGF-IR, ERK1/2, Akt, and paxillin, we used RNA interference by using small-interfering RNA (siRNA). Cells were transfected with vehicle (Diethylpyrocarbonatetreated water), control siRNA (scrambled), or siRNA specific oligos (200 to $400 \mathrm{pmol}$ ) by using the TransIT-siTKO reagent (Mirus Corporation, Madison, WI).
Scramble and anti-IGF-IR siGenome Smartpool siRNA oligos were from Thermo Scientific Dharmacon (Lafayette, CO). Scramble and anti-ERK2 (MAPK1), paxillin, and Akt1 Silencer Select Validated siRNA oligos were from Ambion (Austin, TX). The expression of the IGF-IR and ERK1/2 proteins was detected by immunoblotting by using anti-IGF-IR and anti-ERK1/2 polyclonal antibodies (Santa Cruz Biotechnology). Paxillin was detected by using anti-paxillin monoclonal antibodies (BD Pharmingen, San Diego, CA). Akt was detected by using an anti-Akt polyclonal antibody (Cell Signaling Technology).

\section{Immunofluorescence Studies}

Serum-starved 5637 and T24 cells on coverslip were stimulated for 30 minutes with IGF-I ( $50 \mathrm{ng} / \mathrm{ml})$. Cells were fixed, permeabilized, and blocked as described. ${ }^{20}$ Primary antibodies were as follows: anti-paxillin (BD Transduction Laboratories; 1:100) and anti-Focal Adhesion Kinase (FAK; Invitrogen, Carlsbad, CA; 1:50) antibodies. Secondary antibodies were as follows: Alexa Fluor 488 and 594 (Molecular Probes; Invitrogen). Coverslips were mounted in Vectashield and counterstained with 4',6-diamino-2-phenylindole (DAPI) (Vector Laboratories, Burlingame, CA). Images were analyzed with a LSM 510 Meta Confocal Microscope (objective, ×63; Universal Imaging).
A

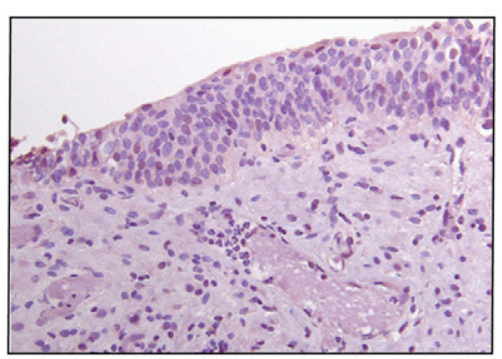

Urothelial Carcinoma

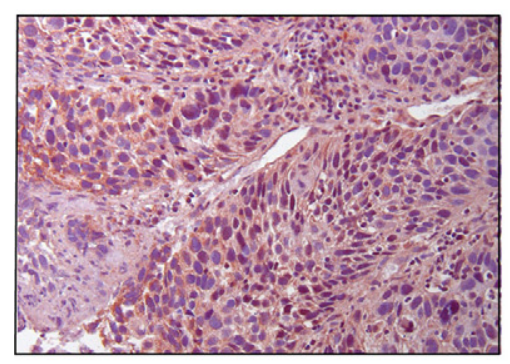

B

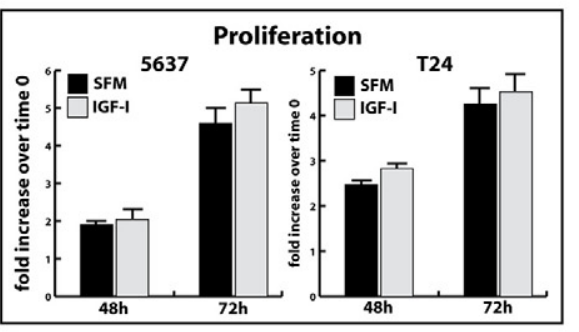

5637

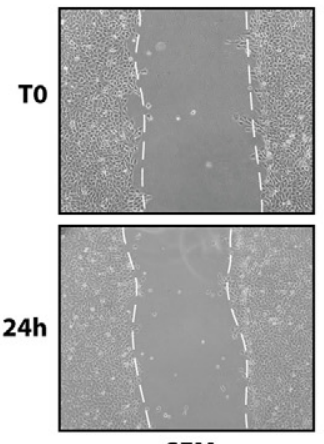

SFM
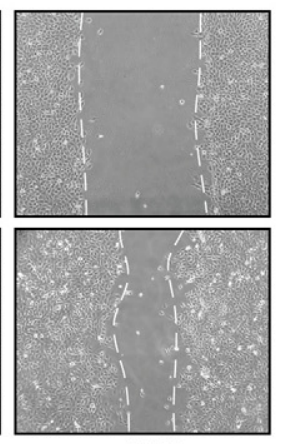

IGF-I
C

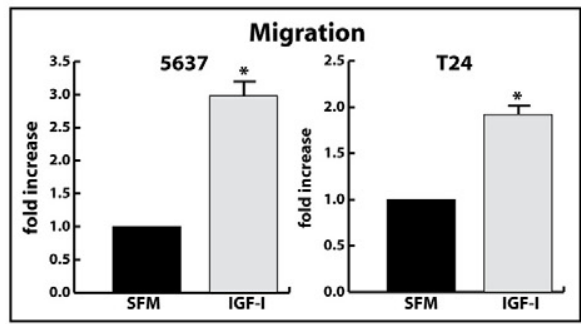

T24
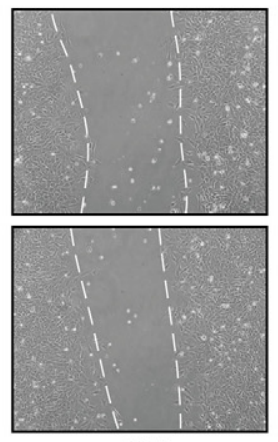

SFM

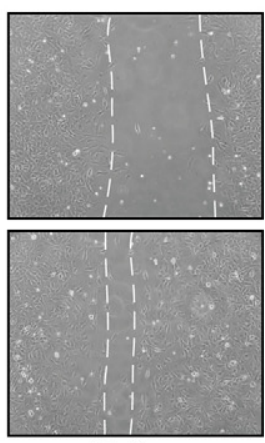

IGF-I

Figure 1. The IGF-IR is overexpressed in invasive bladder cancer and promotes migration and in vitro closure of a wound in urothelial carcinoma-derived cells A: Formalin-fixed paraffin-embedded sections of nonmalignant human bladder and bladder cancer tissue were immunostained by using a polyclonal antibody against human IGF-IR. Top: normal urothelium expressing the IGF-IR (original magnification, $\times 200$ ). Bottom: high levels of IGF-IR in invasive urothelial cancer (original magnification, $\times 200$ ). At least ten independent fields per section were examined. Field is representative of $100 \%$ of fields examined. B and C: cell proliferation and migration assays. Values represent the mean $\pm \mathrm{SD}$ of four independent experiments run in duplicate $\left(\mathbf{C}:{ }^{*} P<0.01\right)$. D: For the wound healing experiments, cells were analyzed with live-cell microscopy by using the Metamorph Image Acquisition and Analysis software (Universal Imaging; original magnification, $\times 100)$. Ten fields per plate were examined. 


\section{Statistical Analysis}

Experiments were performed in triplicate and repeated at least three times. Results are expressed as mean \pm SD. All statistical analyses were performed with SigmaStat for Windows version 3.10 (Systat Software, Inc., Port Richmond, CA). Results were compared by using the twosided Student's t-test. Differences were considered statistically significant at $P<0.05$.

\section{Results}

\section{The IGF-IR Is Overexpressed in Invasive Bladder Cancer}

Recent work by Rochester et $\mathrm{al}^{18}$ has demonstrated by quantitative RT-PCR that IGF-IR mRNA levels were significantly increased in invasive bladder cancer tissues compared with normal urothelium. We determined by immunohistochemical analysis the level of IGF-IR expression in normal and invasive bladder cancer sections. First, we established that the IGF-IR is present, although at low levels, in normal urothelial tissue (Figure 1A, top). Second, we discovered that the IGF-IR was expressed at considerably higher levels in invasive bladder tumor tissues analyzed (Figure 1A, bottom). These results confirm and extend previous reports, which suggest that the IGF-IR may play an important role and may contribute to the invasive phenotype in bladder tumors.

\section{IGF-IR Activation Promotes Migration of Transformed Urothelial Cells Without Affecting Proliferation}

To discern the role of ligand-activated IGF-IR signaling pathway in bladder cancer cells, we first determined the effect of IGF-I on urothelial carcinoma-derived 5637 and T24 cells on cell proliferation and migration. IGF-I did not induce a statistically-significant increase over SFM in mitogenic response in both cell types after either 48 or 72 hours of continuous exposure to the growth factor (Figure 1B). In contrast, IGF-I induced twofold to threefold increases in migration (Figure $1 \mathrm{C}$ ).

Next, we determined the ability of 5637 and T24 bladder cancer cells to migrate in response to IGF-I by using an in vitro "wound healing" motility assay. ${ }^{19-21}$ In contrast to control (Figure 1D, T0 or SFM), IGF-I evoked a substantial migration of both cells into the denuded area (Figure 1D, IGF-I).

These results suggest that the ability of IGF-I to promote migration and lateral motility (wound healing) on cancer urothelial cells can be separated from the capacity to induce cell proliferation.

\section{IGF-IR Activation Enhances Invasiveness}

The acquisition of an invasive phenotype is a critical step for tumor progression. ${ }^{23}$ To this end, we used Matrigelcoated filters to examine invasive migration through a 3D extracellular matrix. ${ }^{20,21}$ After exposure to IGF-I, there was a marked increase in the ability of 5637 and T24 cells to invade a 3D matrix (Figure 2A).

Next, we determined the ability of T24 cells to evade from Matrigel drops. ${ }^{19,22}$ IGF-I stimulation significantly increased the number of T24 cells able to evade the 3D-matrix (Figure 2B). Moreover, the migration distance covered by IGF-I-stimulated T24 cells was markedly increased $(P<0.05)$ as compared with T24 cells incubated with SFM (data not shown).

To confirm that IGF-I-induced migration of 5637 and T24 cells is indeed mediated by the IGF-IR, we depleted endogenous IGF-IR by siRNA approaches. We achieved considerable reduction in the level of endogenous IGF-IR in both 5637 (Figure 2C) and T24 cells (Figure 2D) with a consequent severe inhibition in the ability of these cells to migrate in response to IGF-I stimulation.

Collectively, our results suggest that activation of the IGF-IR by its ligand not only stimulates the migratory ability of urothelial cancer cells, but also the cells' ability to migrate through and from a complex 3D matrix.

\section{Signaling Pathways Stimulated by IGF-I in Bladder Cancer Cells}

To investigate the mechanisms by which the IGF-IR promotes motility and invasion of bladder cancer cells, we first examined the Akt and MAPK pathways, key components for IGF-I-mediated biological responses in many cellular systems. ${ }^{24-26}$ Furthermore, the activation of the MAPK pathway plays a critical role in motility of epithelial cells, as we have recently shown for proepithelin-induced motility of bladder and prostate cancer cells. ${ }^{19-21}$ As determined by immunoblot, IGF-I induced in 5637 cells a sustained activation of Akt and ERK1/2 up to 120 minutes with subsequent activation of downstream effectors S6 ribosomal protein and p90RSK (Figure 3A). Akt and ERK1/2 activation in T24 cells was sustained up to 30 minutes and decreased after 120 minutes of IGF-I stimulation, still remaining above the level of unstimulated cells (Figure 3A). The level of Akt and ERK1/2 proteins was instead not affected by IGF-I stimulation, as determined by immunoblot with anti-Akt and ERK1 antibodies (not shown). Thus, IGF-I-stimulated activation of the Akt and MAPK pathways may be critical for IGF-IR-dependent biological responses in bladder cancer cells.

To corroborate the role of Akt and ERK1/2 activation in IGF-I-mediated responses in urothelial cancer cells, we performed IGF-I-evoked migration and invasion assays in the presence of specific inhibitors of either the Akt (LY294002) or MAPK (U0126) pathways. Notably, incubation with LY294002 and U0126 reduced the ability of 5637 urothelial cells to migrate and invade in response to IGF-I stimulation (Figure 3B), whereas T24 cells were insensitive to MAPK inhibition in invasion (Figure 3C). However, U0126 induced a statistically significant decrease in cell migration (Figure 3C). In T24 cells, the combination of LY294002 and U0126 had stronger effects than the Akt inhibitor alone and significantly inhibited migration and invasion (Figure $3 \mathrm{C}$ ). We verified that 
A

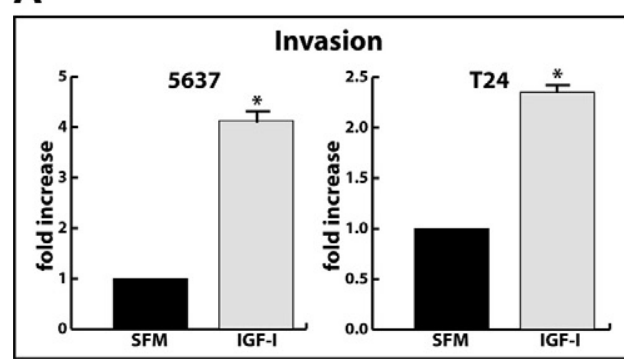

Figure 2. IGF-IR activation promotes invasion and evasion of urothelial carcinoma-derived cells. A: quantification of invading 5637 and T24 cells plated on Matrigelcoated transwells. For 5637 cells, invasion was analyzed at 24 hours, whereas T24 cells were analyzed after eight hours. Data are expressed as fold increase over SFM (normalized to 1). Values represent the mean $\pm \mathrm{SD}$ of three independent experiments run in duplicate $\left({ }^{*} P<0.01\right)$. B: For Matrigel evasion assays, T24 cells were included in a Matrigel drop and allowed to evade for 72 hours. The dotted line indicates the edge of Matrigel drops. Values represent the mean $\pm \mathrm{SD}$ of three independent experiments run in quintuplicate $\left({ }^{*} P<0.05\right)$. C and $\mathbf{D}$ : gene knockdown for the IGF-IR in 5637 and T24 cells was achieved by siRNA. Densitometric analysis is expressed as arbitrary units. One representative blot of two independent experiments is shown. Cells were processed and analyzed for migration as described in Materials and Methods. Values are expressed as fold change over SFM \pm SD. ${ }^{*} P<0.05$ compared with control oligo-treated cells (second columns).
B
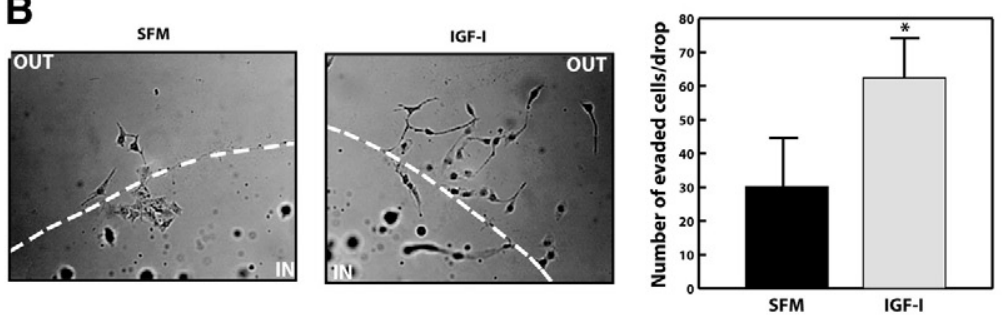

5637

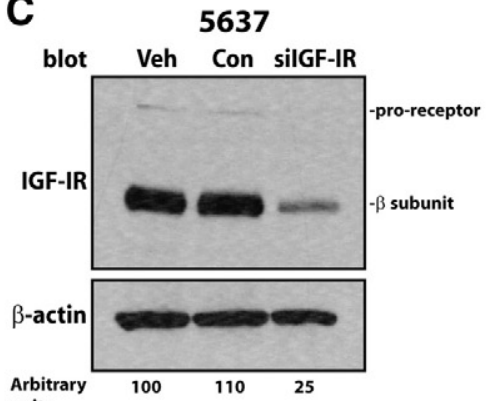

units

D

T24

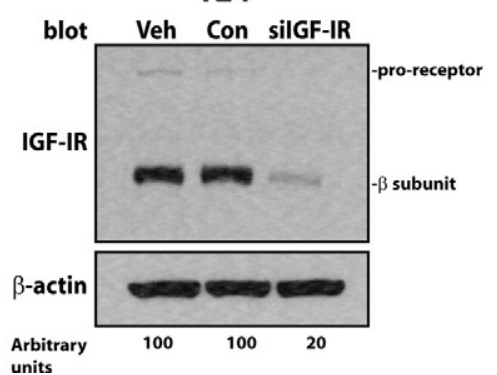

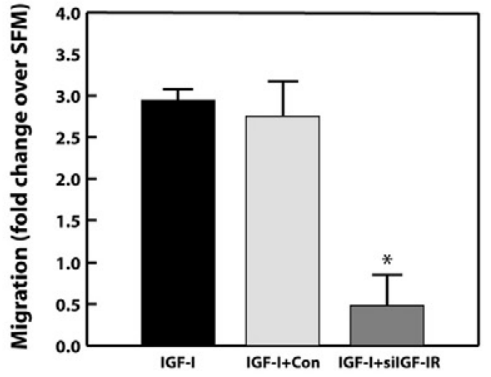

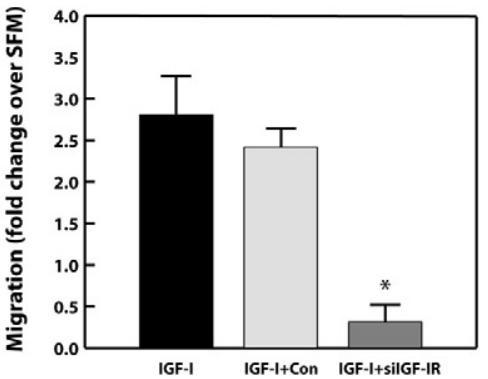

the concentrations of LY294002 and U0126 used for these experiments were effective in inhibiting the activation of Akt and ERK1/2. In 5637 cells, IGF-I-mediated activation of Akt and ERK1/2 was severely reduced in the presence of LY294002 (20 $\mu \mathrm{mol} / \mathrm{L})$ or U0126 (10 $\mu \mathrm{mol} / \mathrm{L})$, respectively, establishing the effectiveness of both inhibitors at the concentration used in migration and invasion assays (data not shown). ${ }^{20,27}$

These results suggest that activation of MAPK and the Akt pathways is necessary for IGF-IR-mediated migration and invasion of urothelial carcinoma-derived cells.

Next, we investigated the role of MAPK and Akt signaling in IGF-I-promoted biological responses in urothelial cancer cells by targeting endogenous Akt and ERK $1 / 2$ proteins using siRNA strategies. Depletion of endogenous ERK2 (Figure 4A) and Akt1 (Figure 4B) significantly reduced the ability of 5637 cells to migrate and invade in response to IGF-I, confirming that the Akt and MAPK pathways are both required for fully sustained IGF-I-induced cell motility of bladder cancer cells.

To rule out the possibility of off target effects, we tested different siRNA oligos for both ERK2 and Akt1 in 5637 cells, and we confirmed that depletion of ERK2 and Akt1 in 5637 cells inhibits IGF-IR-mediated migration of these cells (Supplemental Figure S1, A and B, see http://ajp.amjpathol.org).

Similar to the results obtained with the inhibitors, T24 cells are insensitive to ERK depletion, whereas endoge- nous Akt1 knockdown significantly reduced both migration and invasion in these cells (Supplemental Figure S2, A and B, see http://ajp.amjpathol.org).

\section{IGF-IR-Evoked Paxillin Activation}

Paxillin, with FAK, plays a critical role in cellular motility at focal adhesions. ${ }^{28-30}$ In addition, our recent work has pointed out a role of paxillin in cellular motility induced by the growth factor proepithelin in urothelial cancer cells. ${ }^{20}$

To elucidate in more depth the mechanism(s) by which the IGF-IR promotes motility and invasion of urothelial carcinoma-derived cells, we tested whether IGF-IR activation of Akt and MAPK may promote phosphorylation of paxillin and whether paxillin would be necessary to promote migration and invasion in these cells. We discovered that IGF-I promoted a threefold increase of tyrosine-phosphorylation of paxillin in 5637 cells as compared with unstimulated cells (Figure 5A). Specifically, IGF-I promoted an increase in phosphorylation of Tyrosine 31 compared with unstimulated cells as we determined by using phospho-Tyr-specific antibody and immunoblotting (Figure 5B). We also detected paxillin phosphorylation on Tyrosine 118, but this residue seemed to be constitutively phosphorylated and not modulated by IGF-I in 5637 cells. Significantly, prolonged IGF-I stimulation promoted paxillin phosphorylation on Serine 126 
A

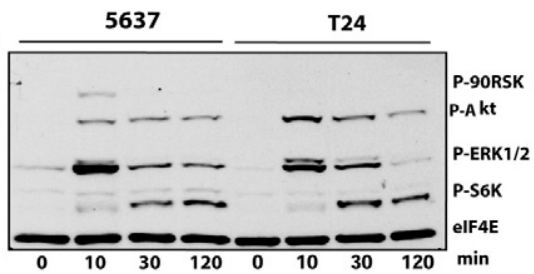

B

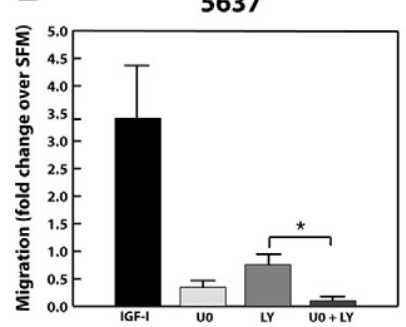

C

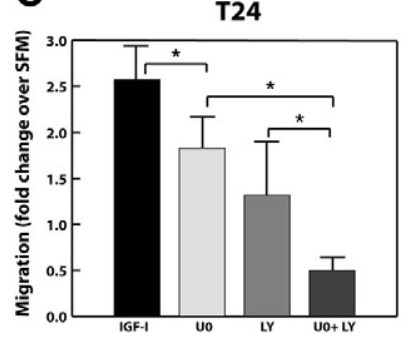

5637

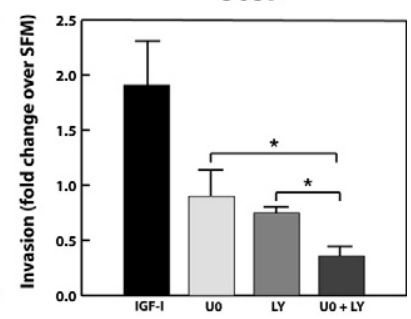

T24

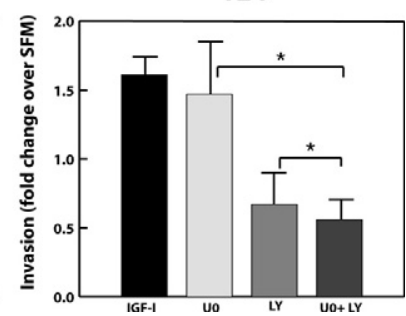

Figure 3. IGF-I regulates the activation of the Akt and MAPK pathway in 5637 and T24 cells. A: The activation of (top to bottom) p90RSK, Akt, ERK1/2, and S6 ribosomal protein was analyzed by immunoblotting by using phosphospecific antibodies. ElF4E protein is the control to monitor the loading of the samples. Blot is representative of three independent experiments. $\mathbf{B}$ and $\mathbf{C}$ : migration and invasion experiments in 5637 and T24 in the presence of IGF-I supplemented or not with inhibitors. Values are expressed as fold change over SFM \pm SD. B: inhibitors alone or in combination; ${ }^{*} P<0.05$ compared with IGF-I alone. C (migration): inhibitors alone or in combination; ${ }^{*} P<0.05$ compared with IGF-I alone. $\mathbf{C}$ (invasion): U0 ${ }^{*} P>0.05$ compared with IGF-I alone. Data are the average of three independent experiments \pm SD run in duplicate.

and Serine 178 (Figure 5B), two possible target sites for Akt and/or ERK-mediated phosphorylation on paxillin. ${ }^{31}$ The same pattern of paxillin phosphorylation induced by IGF-I was detectable in T24 cells (Supplemental Figure S2C, see http://ajp.amjpathol.org).

A

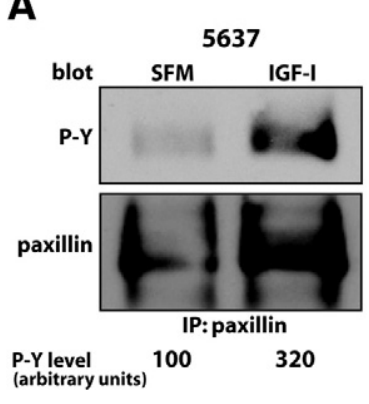

C

5637
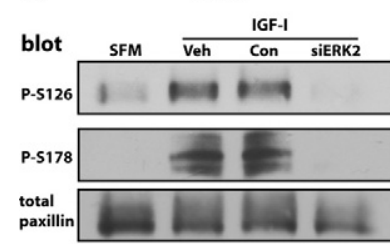

ERKs

in

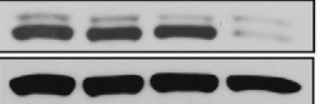

B

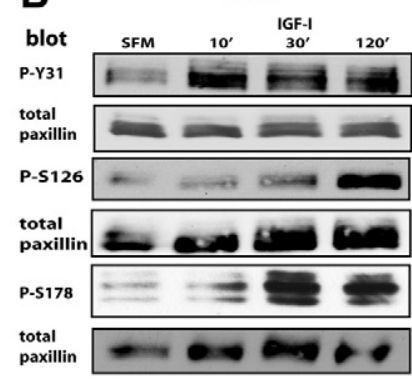

D

5637

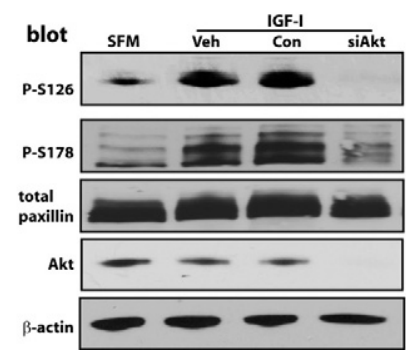

Figure 5. IGF-I induces paxillin phosphorylation. A: lysates $(600 \mu \mathrm{g})$ from 5637 cells were immunoprecipitated with anti-paxillin monoclonal antibodies (BD Pharmingen). Tyrosine-phosphorylated paxillin was detected by immunoblot with anti-phospho-tyrosine-horseradish peroxidase-conjugated monoclonal antibodies (BD Pharmingen). Total paxillin was detected by using anti-paxillin polyclonal antibodies (Millipore, Billerica, MA). The level of phospho-paxillin was normalized over total immunoprecipitated paxillin. The blot is representative of two independent experiments. B: serum-starved 5637 cells were stimulated with IGF-I $(50 \mathrm{ng} / \mathrm{ml})$. Immunoblot was performed by using phosphospecific-paxillin antibodies. Blots are representative of three independent experiments. $\mathbf{C}$ and $\mathbf{D}$ : paxillin phosphorylation was assessed after 2 hours of IGF-I stimulation in ERK-depleted and Aktdepleted 5637 cells as described above. Depletion and detection of endogenous ERK2 and Akt1 was performed as described in Figure 4.

In addition, IGF-I stimulation of 5637 cells induced the formation of a complex that includes phosphorylated paxillin and active ERK1/2, as determined by co-immunoprecipitation and immunoblot experiments (data not shown).

Next, we confirmed that paxillin phosphorylation was regulated by Akt and ERK1/2 by depleting 5637 cells of endogenous ERK2 and Akt1 using siRNA strategies.
A

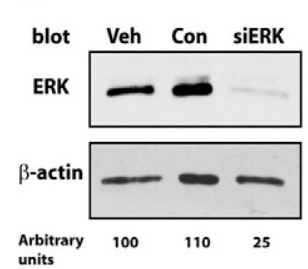

B

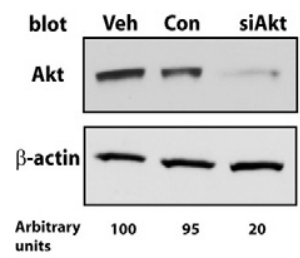

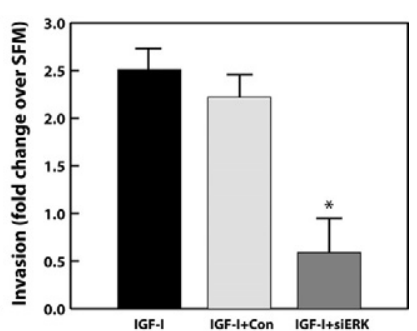

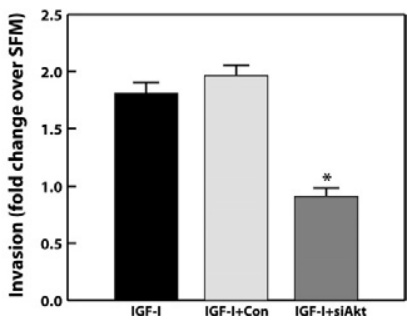

Figure 4. Depletion of endogenous ERK and Akt impair IGF-I-mediated migration and invasion of 5637 cells. A and B: gene knockdown for ERK2 and Akt1 in 5637 cells was achieved by siRNA. The level of endogenous ERK1/2 (A) and Akt (B) proteins was detected by immunoblot by using anti-ERK1/2 (Santa Cruz Biotechnology) or anti-Akt polyclonal antibodies (Cell Signaling Technology). One representative blot of three independent experiments is shown. Migration and invasion is expressed as fold change over $\mathrm{SFM} \pm \mathrm{SD} .{ }^{*} P<0.05$ compared with control oligo-treated cells (second columns). 
A

\section{7}

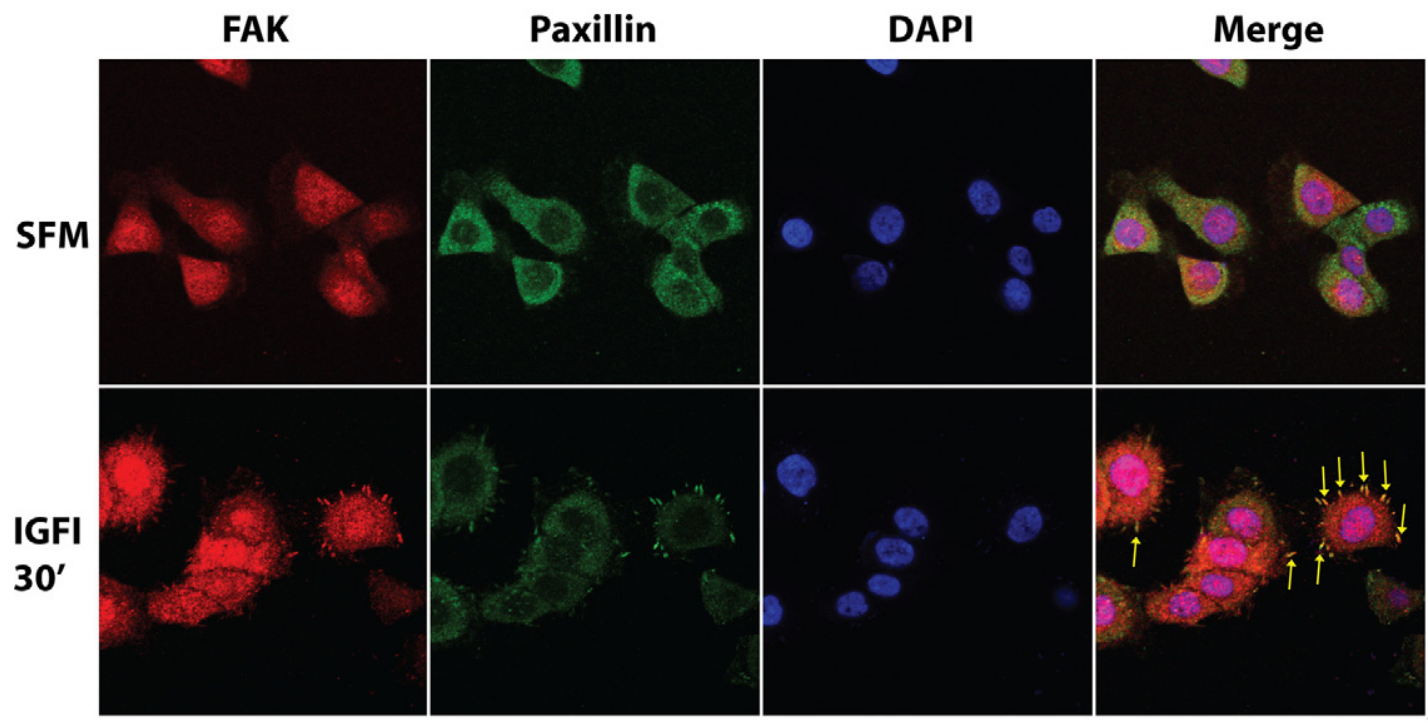

B

T24

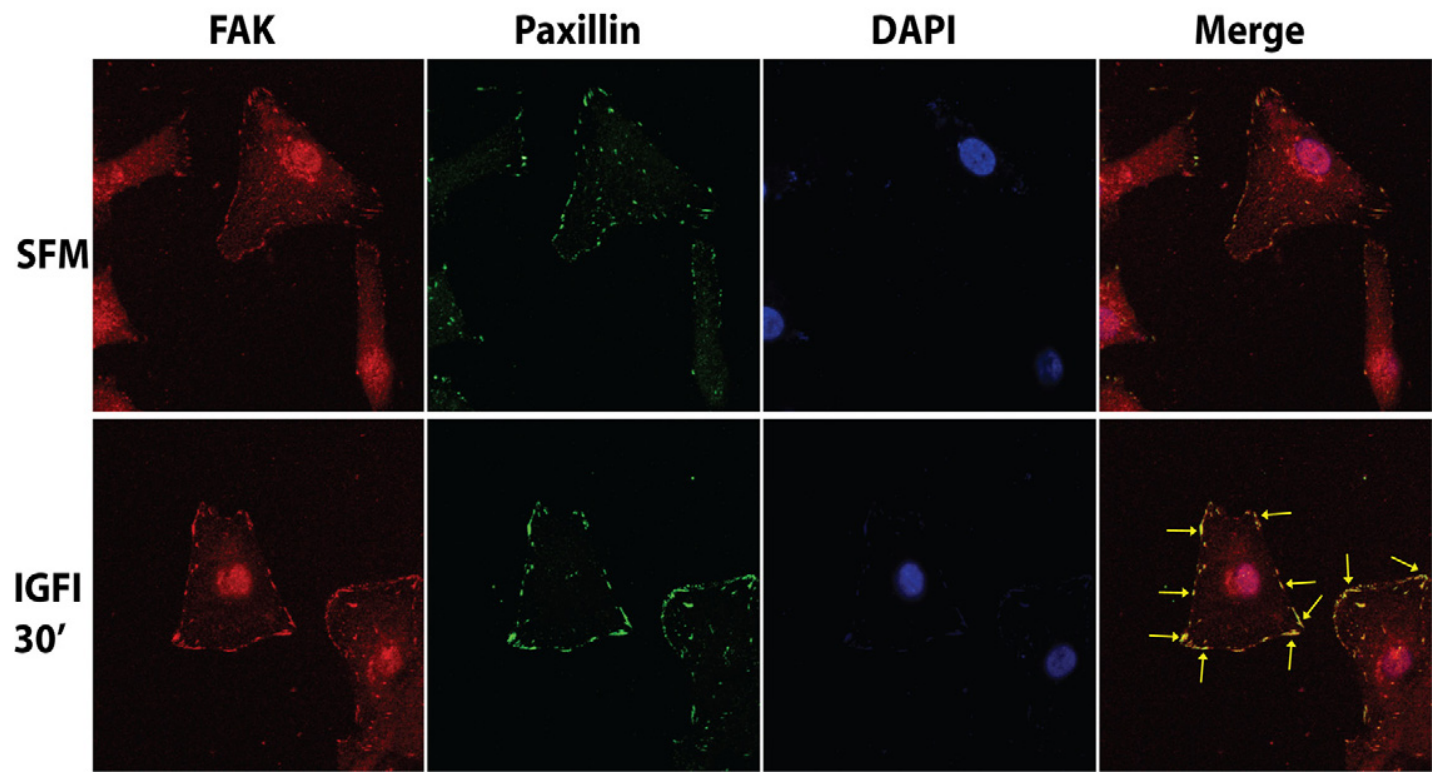

Figure 6. IGF-I promotes paxillin and FAK colocalization in dynamic focal adhesions. A: 5637 and (B) T24 cells were plated onto coverslips, serum-starved for 24 hours, and stimulated for 30 minutes with IGF-I $(50 \mathrm{ng} / \mathrm{ml})$. Colocalization was analyzed by confocal microscopy as described in Materials and Methods. At least 10 independent fields were examined. Images were analyzed at the Kimmel Cancer Center Bioimaging Core Facility with LSM 510 Meta Confocal Microscope by using the Metamorph Image Acquisition and Analysis software (Universal Imaging) using a 63× objective. The images were merged by using Photoshop. Pictures are representative of three independent experiments. Arrows indicate regions where paxillin colocalized with FAK.

ERK2 depletion (Figure 5C) almost completely inhibited paxillin phosphorylation of both Serine 126 and Serine 178, whereas Akt1 knockdown (Figure 5D) completely abolished Serine 126 phosphorylation and reduced phosphorylation of Serine 178.

Collectively, these results indicate that on IGF-I stimulation Akt and MAPK activation regulates paxillin phosphorylation in 5637 urothelial cancer cells. These results also suggest that paxillin may play an important role in mediating IGF-I-induced motility and invasion of bladder cancer cells.

\section{Paxillin Colocalizes with FAK}

The formation and disassembly of focal adhesions (adhesion turnover) at the cell front is a key process in the regulation of cellular migration. ${ }^{32}$ Because paxillin and FAK are key constituents of focal adhesions, ${ }^{32}$ we determined whether IGF-I stimulation could induce a redistribution of paxillin at the cell edge of migrating cells. Thirtyminute stimulation with IGF-I induced a clear change in cellular morphology of 5637 cells and with a significant redistribution of paxillin at the protrusive region in focal 

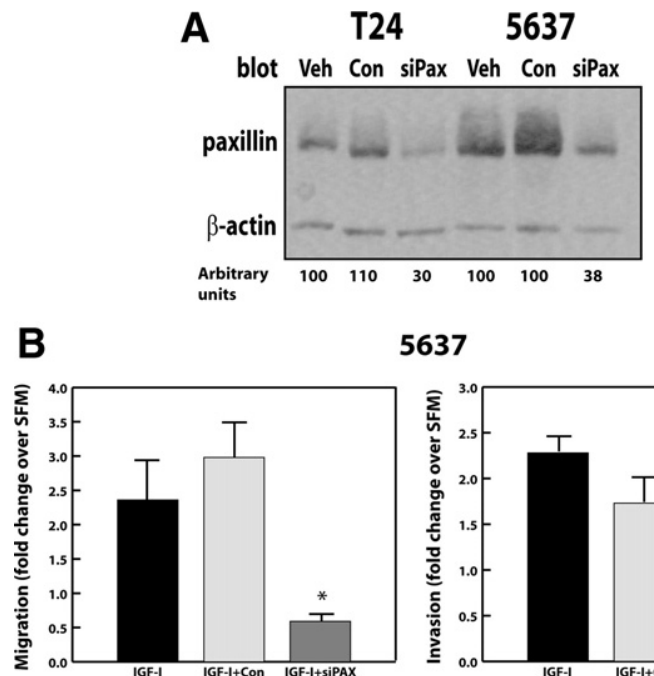

5637

\section{C}
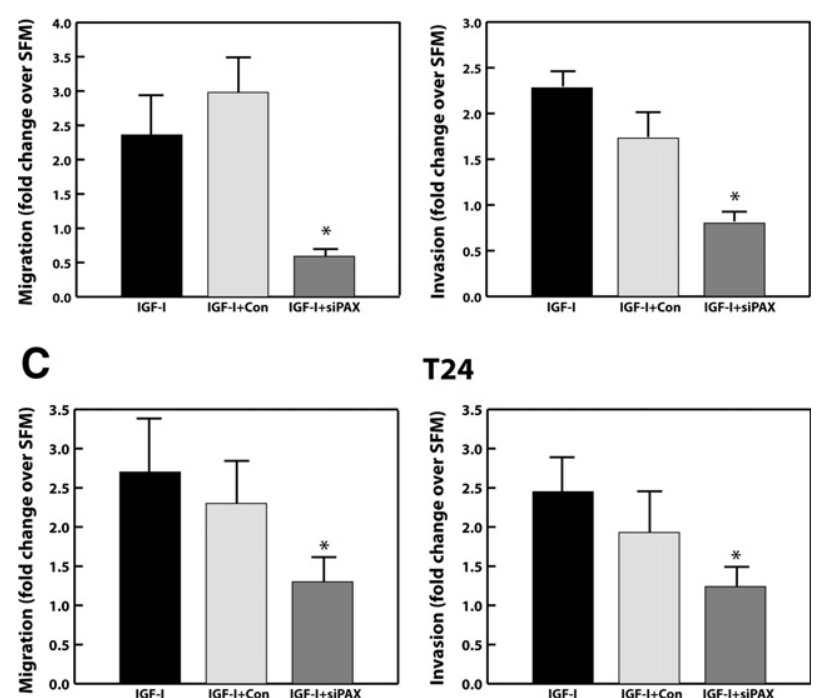

T24

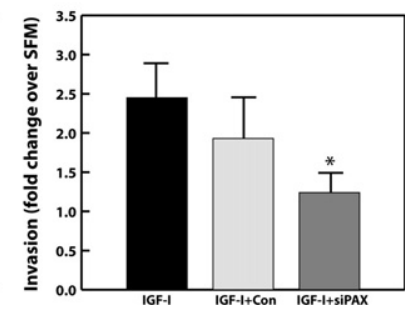

Figure 7. Paxillin depletion inhibits IGF-I-induced migration and invasion of 5637 and T24 urothelial carcinoma-derived cells. A: gene knockdown for paxillin was achieved by RNA interference by using siRNA. Paxillin expression levels were analyzed by immunoblot by using anti-paxillin polyclonal antibodies (Santa Cruz Biotechnology). B: 5637 and T24 (C) cells were processed and analyzed for IGF-I-induced migration and invasion as described in Materials and Methods. Values are expressed as fold change over SFM. ${ }^{*} P<0.05$ compared with control-oligo-transfected cells. Data are the average $\pm \mathrm{SD}$ of three independent experiments in duplicates.

adhesions where paxillin colocalized with FAK (Figure $6 \mathrm{~A}$, arrows). The same redistribution of focal adhesions and colocalization of paxillin with FAK in adhesions was detectable by confocal microscopy in T24 cells but with a slightly higher background in unstimulated cells (Figure 6B, arrows).

Collectively, these results suggest that paxillin may play an important role in IGF-I-induced motility of cancer urothelial cells by regulating adhesion turnover at the cell front, a process critical to cellular migration.

\section{Paxillin Requirement for IGF-IR-Mediated Migration and Invasion}

To confirm the role of paxillin in IGF-IR-induced motility of cancer urothelial cells, we used SiRNA to target endogenous paxillin proteins in 5637 and T24 cells. Our approach yielded a $\sim 70 \%$ depletion of endogenous paxillin proteins in both 5637 and T24 cells as compared with cells treated with either vehicle or scrambled siRNA (Figure 7A) and a robust inhibition of IGF-I-mediated migration and invasion of 5637 (Figure 7B) and T24 (Figure 7C) cells.
Collectively, our findings reveal an essential role for paxillin in the IGF-IR functional regulation of tumor cell motility, a key property of the aggressive cancer phenotype.

\section{Discussion}

Although bladder cancer is one of the most common malignancies, ${ }^{1}$ the molecular mechanisms that determine malignant transformation in the urothelia lining the bladder wall are still very poorly characterized. Most bladder cancers are frequently recurring and often progress into an invasive and metastatic phenotype, regardless of treatment with surgery, chemotherapy, or immunotherapy. The present study provides the first evidence that the activation of the IGF-IR pathway might play a significant role in bladder tumor formation and progression by promoting migration and invasion of bladder cancer cells.

Because the IGF-IR is overexpressed in invasive bladder cancer, we used urothelial carcinoma-derived human 5637 and T24 cells to determine the role of the IGF-IR, and we show the following: (1) activation of the IGF-IR by its ligand IGF-I promotes migration and induces wound healing in these cells without affecting cell proliferation; (2) the IGF-IR stimulates the cells' ability to migrate through and from a complex 3D matrix such as Matrigel; (3) IGF-I stimulation induces the activation of both the Akt and MAPK pathways, which are required for migration and invasion, as determined by pharmacological and genetic approaches; (4) IGF-I stimulation induces Akt and MAPK-dependent paxillin phosphorylation; (5) on IGF-I stimulation, paxillin relocates at dynamic focal adhesion at the cell's edge where it colocalizes with FAK; and (6) depletion of endogenous paxillin proteins by siRNA strategies severely reduces motility and invasive ability of these cells.

The IGF-IR plays a critical role in the regulation of cell growth $^{4,5}$ and it is essential for transformation for its ability to protect cancer cells from apoptosis. ${ }^{12-15}$ The IGF-IR has been implicated in promoting cellular motility of breast cancer cells, ${ }^{33,34}$ and the enhancement of cell migration mediated by the activation of the IGF-IR has been directly linked to the progression toward a metastatic phenotype. ${ }^{35,36}$ In addition, the expression of a constitutively active IGF-IR causes transformation and xenograft growth of immortalized mammary epithelial cells and is accompanied by an epithelial-to-mesenchymal transition. ${ }^{37}$

The IGF-IR is now accepted as a very attractive candidate for cancer therapy. There are currently approximately 12 anti-IGF-IR therapeutic agents undergoing clinical evaluation, including blocking antibodies and tyrosine kinase inhibitors (small molecules). The complete list of anti-IGF-IR therapeutics is summarized in a recent review by Chitnis et al. ${ }^{16}$

A definite role for the IGF-IR in transformation of urothelial cells is not clearly established, but Rochester et al ${ }^{18}$ recently reported that the IGF-IR is up-regulated in bladder cancer tissues compared with nonmalignant tissue controls. They also showed by quantitative real-time poly- 
merase chain reaction (RT-PCR) that IGF-IR mRNA levels were significantly increased in invasive cancers compared with normal urothelium. ${ }^{18}$ We have confirmed the results of the IGF-IR mRNA analysis by demonstrating that the IGF-IR is indeed overexpressed in invasive bladder cancer tissues compared with normal counterpart. Our results strongly support the hypothesis that the IGF-IR might contribute to the transition to the invasive phenotype in bladder cancer by providing the first evidence that the activation of the IGF-IR strongly promotes migration, lateral motility, and invasion through extracellular matrix of urothelial cancer cells.

We have examined the signaling pathways activated by IGF-I in urothelial carcinoma-derived cell lines and have shown that Akt and ERK $1 / 2$ activation is required for IGF-I-induced motility and invasion of 5637 and T24 human bladder cancer cells. Compared with 5637 cells, T24 cells are quite insensitive to pharmacological and genetic ablation of ERK1/2, but the combination of Akt and MAPK pharmacological inhibitors had a stronger effect than the Akt inhibitor alone in these cells. These results suggest that in T24 cells the Akt pathway may play a more critical role than ERK $1 / 2$ in promoting migration and invasion in these cells. T24 cells express an activated Ras protein, ${ }^{38}$ which may sustain a signal that would promote motility and invasion in ERK-independent fashion.

Our study identified paxillin as an essential component of IGF-I-stimulated signaling leading to motility and invasion of T24 and 5637 cells. On IGF-I stimulation, paxillin is phosphorylated in Akt and ERK-dependent fashion, colocalizes with FAK at dynamic focal adhesion at the cells edge of migrating cells, and it is necessary for motility and invasion as paxillin knockdown severely reduced the ability of 5637 and T24 cells to migrate and invade.

Our results do not demonstrate that paxillin is a direct substrate of Akt and ERK1/2 phosphorylation but clearly establish that Akt and ERK1/2 play a critical role in regulating IGF-I-mediated paxillin phosphorylation. As previously demonstrated in macrophages, paxillin phosphorylation by Glycogen-Synthase Kinase 3 (GSK-3) is regulated via an ERK-dependent priming mechanism ${ }^{31}$; our results would support a similar scenario in 5637 and T24 cells.

We have identified Serine 126 and Serine 178 as phosphorylation sites induced by IGF-I in 5637 and T24 cells suggesting that these residues may play an important role in regulating IGF-I-dependent motility and invasion. However, our experiments do not demonstrate that these residues are absolutely required for paxillin activation, and we do not rule out that additional residues may be phosphorylated on IGF-I stimulation and may contribute to paxillin function in IGF-I-dependent motility and invasion.

IGF-I stimulation induced paxillin phosphorylation at Tyrosine 31, which is mediated by FAK in other cellular models ${ }^{39}$ suggesting that IGF-I stimulation may induce FAK-dependent paxillin phosphorylation.

FAK activation links growth factors and integrin signaling pathways and has an important function in promoting growth factors and integrin-mediated cell migration and survival. ${ }^{39}$ FAK is required for PI3K- and Ras-dependent breast tumorigenesis, ${ }^{40}$ and integrins through FAK activate Ras signaling to MAPK. ${ }^{23,41}$ Thus, a plausible mechanism by which IGF-I promotes migration and invasion of bladder cancer cells would be by activating the FAK/ integrins complex and the signaling cascade leading to Akt and MAPK activation thereby promoting Akt and MAPK-dependent paxillin phosphorylation.

In conclusion, the IGF-IR as a critical regulator of tumor cell motility, and invasion of bladder cancer cells could represent a novel molecular target in bladder cancer. The IGF-IR could also serve as a novel tumor biomarker for diagnosis and possibly prognosis of bladder tumors.

\section{References}

1. Jemal A, Siegel R, Ward E, Hao Y, Xu J, Thun MJ: Cancer statistics, 2009. CA Cancer J Clin 2009, 59:225-249

2. Knowles MA: Molecular pathogenesis of bladder cancer. Int J Clin Oncol 2008, 13:287-297

3. Mitra AP, Cote RJ: Molecular pathogenesis and diagnostics of bladder cancer. Annu Rev Pathol 2009, 4:251-285

4. Scher CD, Stone ME, Stiles CD: Platelet-derived growth factor prevents G0 growth arrest. Nature 1979, 281:390-392

5. Baserga R: The insulin-like growth factor I receptor: a key to tumor growth? Cancer Res 1995, 55:249-252

6. Baker J, Liu JP, Robertson EJ, Efstratiadis A: Role of insulin-like growth factors in embryonic and postnatal growth. Cell 1993 75:73-82

7. Eggenschwiler J, Ludwig T, Fisher P, Leighton PA, Tilghman SM, Efstratiadis A: Mouse mutant embryos overexpressing IGF-II exhibit phenotypic features of the Beckwith-Wiedemann and Simpson-Golabi-Behmel syndromes. Genes Dev 1997, 11:3128-3142

8. Surmacz E: Growth factor receptors as therapeutic targets: strategies to inhibit the insulin-like growth factor I receptor. Oncogene 2003, 22:6589-6597

9. Baserga R, Morrione A: Differentiation and malignant transformation: two roads diverged in a wood. J Cell Biochem 1999, Suppl 32-33: 68-75

10. Pollak MN, Schernhammer ES, Hankinson SE: Insulin-like growth factors and neoplasia. Nat Rev Cancer 2004, 4:505-518

11. Le Roith D, Karas M, Yakar S, Qu BH, Wu Y, Blakesley VA: The role of the insulin-like growth factors in cancer. Isr Med Assoc J 1999, 1:25-30

12. Cheng MC, Lin CH, Lin HJ, Yu YP, Wu SH: Hydrolysis, lactonization, and identification of alpha(2->8)/alpha(2->9) alternatively linked tri-, tetra-, and polysialic acids. Glycobiology 2004, 14:147-155

13. Baserga R: The contradictions of the insulin-like growth factor 1 receptor. Oncogene 2000, 19:5574-5581

14. Baserga R, Hongo A, Rubini M, Prisco M, Valentinis B: The IGF-I receptor in cell growth, transformation and apoptosis. Biochim Biophys Acta 1997, 1332:F105-F126

15. Le Roith D: Regulation of proliferation and apoptosis by the insulinlike growth factor I receptor. Growth Horm IGF Res 2000, 10(Suppl A):S12-S13

16. Chitnis MM, Yuen JS, Protheroe AS, Pollak M, Macaulay VM: The type 1 insulin-like growth factor receptor pathway. Clin Cancer Res 2008, 14:6364-6370

17. Rowinsky EK, Youssoufian H, Tonra JR, Solomon P, Burtrum D, Ludwig DL: IMC-A12, a human IgG1 monoclonal antibody to the insulinlike growth factor I receptor. Clin Cancer Res 2007, 13:5549S-5555S

18. Rochester MA, Patel N, Turney BW, Davies DR, Roberts IS, Crew J, Protheroe A, Macaulay VM: The type 1 insulin-like growth factor receptor is over-expressed in bladder cancer. BJU Int 2007, 100:1396-1401

19. Lovat F, Bitto A, Xu SQ, Fassan M, Goldoni S, Metalli D, Wubah V, McCue P, Serrero G, Gomella LG, Baffa R, lozzo RV, Morrione A: Proepithelin is an autocrine growth factor for bladder cancer. Carcinogenesis 2009, 30:861-868

20. Monami G, Gonzalez EM, Hellman M, Gomella LG, Baffa R, Iozzo RV, Morrione A: Proepithelin promotes migration and invasion of 5637 
bladder cancer cells through the activation of ERK $1 / 2$ and the formation of a paxillin/FAK/ERK complex. Cancer Res 2006, 66:7103-7110

21. Monami G, Emiliozzi V, Bitto A, Lovat F, Xu SQ, Goldoni S, Fassan M, Serrero G, Gomella LG, Baffa R, lozzo RV, Morrione A: Proepithelin regulates prostate cancer cell biology by promoting cell growth, migration, and anchorage-independent growth. Am J Pathol 2009, 174:1037-1047

22. Belletti B, Nicoloso MS, Schiappacassi M, Berton S, Lovat F, Wolf K, Canzonieri V, D'Andrea S, Zucchetto A, Friedl P, Colombatti A, Baldassarre G: Stathmin activity influences sarcoma cell shape, motility, and metastatic potential. Mol Biol Cell 2008, 19:2003-2013

23. Guo W, Giancotti FG: Integrin signalling during tumour progression. Nat Rev Mol Cell Biol 2004, 5:816-826

24. Morrione A, Romano G, Navarro M, Reiss K, Valentinis B, Dews M, Eves E, Rosner MR, Baserga R: Insulin-like growth factor I receptor signaling in differentiation of neuronal H19-7 cells. Cancer Res 2000, 60:2263-2272

25. Peruzzi F, Prisco M, Dews M, Salomoni P, Grassilli E, Romano G, Calabretta B, Baserga R: Multiple signaling pathways of the insulinlike growth factor 1 receptor in protection from apoptosis. Mol Cell Biol 1999, 19:7203-7215

26. Peruzzi F, Prisco M, Morrione A, Valentinis B, Baserga R: Anti-apoptotic signaling of the insulin-like growth factor-I receptor through mitochondrial translocation of c-Raf and Nedd4. J Biol Chem 2001, 276:25990-25996

27. Monami G, Emiliozzi V, Morrione A: Grb10/Nedd4-mediated multiubiquitination of the insulin-like growth factor receptor regulates receptor internalization. J Cell Physiol 2008, 216:426-437

28. Ishibe S, Joly D, Liu ZX, Cantley LG: Paxillin serves as an ERKregulated scaffold for coordinating FAK and Rac activation in epithelial morphogenesis. Mol Cell 2004, 16:257-267

29. Ishibe S, Joly D, Zhu X, Cantley LG: Phosphorylation-dependent paxillin-ERK association mediates hepatocyte growth factor-stimulated epithelial morphogenesis. Mol Cell 2003, 12:1275-1285

30. Petit V, Boyer B, Lentz D, Turner CE, Thiery JP, Valles AM: Phosphorylation of tyrosine residues 31 and 118 on paxillin regulates cell migration through an association with CRK in NBT-II cells. J Cell Biol 2000, 148:957-970

31. Cai X, Li M, Vrana J, Schaller MD: Glycogen synthase kinase 3- and extracellular signal-regulated kinase-dependent phosphorylation of paxillin regulates cytoskeletal rearrangement. Mol Cell Biol 2006, 26:2857-2868

32. Webb DJ, Donais K, Whitmore LA, Thomas SM, Turner CE, Parsons JT, Horwitz AF: FAK-Src signalling through paxillin, ERK, and MLCK regulates adhesion disassembly. Nat Cell Biol 2004, 6:154-161

33. Guvakova MA, Surmacz E: Overexpressed IGF-I receptors reduce estrogen growth requirements, enhance survival, and promote $\mathrm{E}$ cadherin-mediated cell-cell adhesion in human breast cancer cells. Exp Cell Res 1997, 231:149-162

34. Mauro L, Sisci D, Bartucci M, Salerno M, Kim J, Tam T, Guvakova MA, Ando S, Surmacz E: SHC-alpha5beta1 integrin interactions regulate breast cancer cell adhesion and motility. Exp Cell Res 1999, 252:439-448

35. Doerr ME, Jones Jl: The roles of integrins and extracellular matrix proteins in the insulin-like growth factor I-stimulated chemotaxis of human breast cancer cells. J Biol Chem 1996, 271:2443-2447

36. Bartucci M, Morelli C, Mauro L, Ando S, Surmacz E: Differential insulin-like growth factor I receptor signaling and function in estrogen receptor (ER)-positive MCF-7 and ER-negative MDA-MB-231 breast cancer cells. Cancer Res 2001, 61:6747-6754

37. Kim HJ, Litzenburger BC, Cui X, Delgado DA, Grabiner BC, Lin X, Lewis MT, Gottardis MM, Wong TW, Attar RM, Carboni JM, Lee AV: Constitutively active type I insulin-like growth factor receptor causes transformation and xenograft growth of immortalized mammary epithelial cells and is accompanied by an epithelial-to-mesenchymal transition mediated by NF-kappaB and snail. Mol Cell Biol 2007, 27:3165-3175

38. Manning DL, Daly RJ, Lord PG, Kelly KF, Green CD: Effects of oestrogen on the expression of a $4.4 \mathrm{~kb}$ mRNA in the ZR-75-1 human breast cancer cell line. Mol Cell Endocrinol 1988, 59:205-212

39. Zhao J, Guan JL: Signal transduction by focal adhesion kinase in cancer. Cancer Metastasis Rev 2009, 28:35-49

40. Pylayeva Y, Gillen KM, Gerald W, Beggs HE, Reichardt LF, Giancotti FG: Ras- and PI3K-dependent breast tumorigenesis in mice and humans requires focal adhesion kinase signaling. J Clin Invest 2009 119:252-266

41. Bao W, Stromblad S: Integrin alphav-mediated inactivation of p53 controls a MEK1-dependent melanoma cell survival pathway in threedimensional collagen. J Cell Biol 2004, 167:745-756 